\title{
A NOTE ON INTERPOLATION OF SEMIGROUPS
}

\author{
V. KÉYANTUO
}

(Communicated by Palle E. T. Jorgensen)

\begin{abstract}
In this note, we extend the theory of interpolation of semigroups considered by Arendt (1991) and Arendt-Neubrander-Schlotterbeck (1992) to include the fractional order case. We then apply the results to the Schrödinger and wave equations on $L^{p}(\Omega)$ where $\Omega \subset \mathbb{R}^{N}$ is open with bounded Lebesgue measure.
\end{abstract}

\section{INTRODUCTION}

Let $X$ be a Banach space and $A: D(A) \subset X \rightarrow X$ a closed linear operator. Let $E$ be another Banach space. We shall use the notation $E \hookrightarrow X$ to indicate that $E$ is continuously imbedded in $X$. If $E$ is such that $E \hookrightarrow X$, we denote by $A_{E}$ the part of $A$ in $E$, that is, the operator defined by

$$
D\left(A_{E}\right):=\{x \in D(A) \cap E, A x \in E\} \text { and } A_{E} x=A x \text { for } x \in D\left(A_{E}\right) \text {. }
$$

It is clear that $A_{E}$ is also closed. Note that if $D(A) \subset E$, then one has automatically $D(A) \hookrightarrow E$ as a consequence of the closed graph theorem, where $D(A)$ is endowed with the graph norm. One can easily prove the following propositions.

Proposition 1.1. Let $(T(t))$ be a $C_{0}$-semigroup with generator $A$. If $\Phi$ is an interpolation functor, then the part of $A$ in $\Phi(D(A) ; X)$ is the generator of a $C_{0}$-semigroup.

Proposition 1.2. Let $(T(t))$ be a uniformly bounded $C_{0}$-semigroup with generator $A$. We assume that $0 \in \rho(A)$. Then for $\alpha>0$, the part of $A$ in $D\left((-A)^{\alpha}\right)$ is the generator of a $C_{0}$-semigroup.

We remark that the restrictions on $(T(t))$ in Proposition 1.2 cause no difficulty because the class of $C_{0}$-semigroup generators is stable under bounded perturbations.

We now assume that $D\left(A^{n}\right) \subset E \hookrightarrow X$ where $n \in \mathbb{N}$; and if $n>1$, we assume further that there exists $\lambda_{0} \in \rho(A)$ such that $R\left(\lambda_{0}, A\right) E \subset E$ (this implies the same property for all $\lambda \in \rho(A))$. Then in Arendt, Neubrander, and Schlotterbeck [5] it was proved that $A_{E}$ is the generator of an $n$-times integrated semigroup. The simple example $X=L^{2}(0,1), A=i \Delta$ with Dirichlet or Neumann boundary conditions, and $E=L^{\infty}(0,1)$ shows that in general, $A_{E}$

Received by the editors August 18, 1993 and, in revised form, October 22, 1993. 1991 Mathematics Subject Classification. Primary 47D06, 47F05. 
is not a $C_{0}$-semigroup generator. Actually, in this example, the domain of $A_{E}$ is not dense.

In $\S 2$ we prove that if $\alpha>0$ and $E$ is a Banach space such that $D\left((-A)^{\alpha}\right) \subset$ $E \hookrightarrow X$ (with $R\left(\lambda_{0}, A\right) E \subset E$ for some $\lambda_{0} \in \rho(A)$ if $\alpha>1$ ), then $A_{E}$ generates a $\beta$-times integrated semigroup whenever $\beta>\alpha$. Of course if $\alpha \in$ $\mathbb{N}$, by the result of [5] mentioned above, $A_{E}$ generates an $\alpha$-times integrated semigroup ( $\S 2)$. Similar results are derived for cosine functions ( $\S 3)$. In $\S 4$, application to elliptic operators on $L^{p}(\Omega)$, where $\Omega \subset R^{N}$ is an open subset with finite Lebesgue measure, enables us to make precise the results of [2] on the Schrödinger equation.

\section{INTERPOLATION OF SEMIGROUPS}

As announced in the introduction, we shall use the theory of fractional powers of linear operators (see Komatsu [16] for details on this subject) to obtain interpolation results for semigroups. The corresponding results for cosine functions will be considered in the next section.

We first recall some definitions concerning integrated semigroups and integrated cosine functions.

Definition 2.1. Let $\alpha \geq 0$. Then $A$ is the generator of an $\alpha$-times integrated semigroup if $(\omega, \infty) \subset \rho(A)$ for some $\omega \in \mathbb{R}$ and there exists a strongly continuous mapping $S:[0, \infty) \rightarrow \mathscr{L}(X)$ with $\|S(t)\| \leq M e^{\omega t}, t \geq 0$, for some $M \geq 0$ such that

$$
R(\lambda, A)=\lambda^{\alpha} \int_{0}^{\infty} e^{-\lambda t} S(t) d t \text { for } \lambda>\max \{\omega, 0\}
$$
A.

In this case, $(S(t))$ is called the $\alpha$-times integrated semigroup generated by

If $\alpha=0$, then $(S(t))$ is a $C_{0}$-semigroup generated by $A$. It follows from the uniqueness theorem for the Laplace transform that when such a family $(S(t))$ exists, it is unique. The same remark applies to Definition 2.2 below. It is also worth noting that if an operator $A$ generates a $C_{0}$-semigroup $(T(t))$, then for any $\alpha \geq 0, A$ generates an $\alpha$-times integrated semigroup $\left(S_{\alpha}(t)\right)$ which is just $S_{\alpha}(t)=\Gamma(\alpha)^{-1} \int_{0}^{t}(t-s)^{\alpha-1} T(s) x d s, t \geq 0$. The converse statement is not true (see, e.g., [1, 13, and 17] for relevant examples).

For the relationship between $\alpha$-times integrated semigroups and the Cauchy problem, see [1] or [13]. A similar notion exists and is related to the secondorder Cauchy problem.

Definition 2.2. Let $\alpha \geq 0$. Then $A$ is the generator of an $\alpha$-times integrated cosine function if there exist $M, \omega \in \mathbb{R}$ such that $\left\{\lambda: \lambda^{2}>\omega\right\} \subset \rho(A)$ and there exists a strongly continuous mapping $S:[0, \infty) \rightarrow \mathscr{L}(X)$ with $\|S(t)\| \leq$ $M e^{\omega t}, t \geq 0$, such that

$$
R\left(\lambda^{2}, A\right)=\lambda^{\alpha-1} \int_{0}^{\infty} e^{-\lambda t} S(t) d t \text { for } \lambda>\max \{\omega, 0\}
$$

As for the case of Definition 2.1, $(S(t))$ is the $\alpha$-times integrated cosine function generated by $A$. Similarly, for $\alpha=0,(S(t))$ is the strongly continuous cosine function generated by $A$. The sine function corresponds to the case 
$\alpha=1$. For fundamental facts about cosine function theory one may consult Fattorini [10]. For integrated cosine functions, see [4] or [13].

In order to prove the interpolation result for semigroups we need the following result from Butzer and Berens [6, p. 156], characterizing the domain of the fractional power of the infinitesimal generator of a uniformly bounded $C_{0}$-semigroup.

Proposition 2.3. Let $A$ be the generator of a uniformly bounded semigroup $(T(t))$ in $X$ and $0<\alpha<1$. Then for $x \in X$ we have

$$
x \in D\left((-A)^{\alpha}\right) \Leftrightarrow s-\lim _{\varepsilon \rightarrow 0} \frac{1}{\Gamma(-\alpha)} \int_{\varepsilon}^{\infty} t^{-\alpha-1}(T(t)-I) x d t \text { exists. }
$$

In this case, $(-A)^{\alpha} x=-s-\lim _{\varepsilon \rightarrow 0} \frac{1}{\Gamma(-\alpha)} \int_{\varepsilon}^{\infty} t^{-\alpha-1}(T(t)-I) x d t$.

From this we prove the following lemma.

Lemma 2.4. Let $\tau \geq 0, x \in X$. We put $U_{\beta}(\tau) x:=\int_{0}^{\tau}(\tau-s)^{\beta-1} T(s) x d s$. Then if $0<\alpha<\beta \leq 1, U_{\beta}(\tau) x \in D\left((-A)^{\alpha}\right)$.

Proof. Let $t>0$. Then

$$
\begin{aligned}
(T(t)-I) U_{\beta}(\tau) x & =(T(t)-I) \int_{0}^{\tau}(\tau-s)^{\beta-1} T(s) x d s \\
& =\int_{0}^{\tau}(\tau-s)^{\beta-1} T(t+s) x d s-\int_{0}^{\tau}(\tau-s)^{\beta-1} T(s) x d s \\
& =\int_{t}^{t+\tau}(t+\tau-s)^{\beta-1} T(s) x d s-\int_{0}^{\tau}(\tau-s)^{\beta-1} T(s) x d s .
\end{aligned}
$$

We prove that near the origin the following estimate holds:

$$
\mathscr{I}:=\left\|\int_{t}^{t+\tau}(t+\tau-s)^{\beta-1} T(s) x d s-\int_{0}^{\tau}(\tau-s)^{\beta-1} T(s) x d s\right\|=O\left(t^{\beta}\right)
$$

In fact, set $M:=\sup _{t \geq 0}\|T(t) x\|$. From the simple observation that

$$
\begin{aligned}
\int_{t}^{t+\tau} & (t+\tau-s)^{\beta-1} T(s) x d s \\
= & \int_{\tau}^{t+\tau}(t+\tau-s)^{\beta-1} T(s) x d s+\int_{0}^{\tau}(t+\tau-s)^{\beta-1} T(s) x d s \\
& -\int_{0}^{t}(t+\tau-s)^{\beta-1} T(s) x d s,
\end{aligned}
$$


we have

$$
\begin{aligned}
\mathscr{I}= & \| \int_{0}^{\tau}\left[(t+\tau-s)^{\beta-1}-(\tau-s)^{\beta-1}\right] T(s) x d s+\int_{\tau}^{t+\tau}(t+\tau-s)^{\beta-1} T(s) x d s \\
\leq & M\left\{\int_{0}^{\tau}\left|(\tau-s)^{\beta-1}-(t+\tau-s)^{\beta-1}\right| d s\right. \\
& \left.\quad+\int_{\tau}^{t+\tau}(t+\tau-s)^{\beta-1} d s+\int_{0}^{t}(t+\tau-s)^{\beta-1} d s\right\} \\
\leq & M\left\{\int_{0}^{\tau}\left[(\tau-s)^{\beta-1}-(t+\tau-s)^{\beta-1}\right] d s\right. \\
& \left.\quad+\int_{\tau}^{t+\tau}(t+\tau-s)^{\beta-1} d s+\int_{0}^{t}(t+\tau-s)^{\beta-1} d s\right\} \\
= & -\frac{M}{\beta}\left\{\left[(\tau-s)^{\beta}-(t+\tau-s)^{\beta}\right]_{0}^{\tau}+\left[(t+\tau-s)^{\beta}\right]_{\tau}^{t+\tau}+\left[(t+\tau-s)^{\beta}\right]_{0}^{t}\right\} \\
= & \frac{2 M}{\beta} t^{\beta} .
\end{aligned}
$$

This ensures the convergence of the integral in Proposition 2.3 near the origin. The convergence at infinity poses no difficulty.

Remark. The following is well known. $x \in X \Rightarrow \int_{0}^{t} T(s) x d s \in D(A)$ and $A \int_{0}^{t} T(s) x d s=T(t) x-x$. Hence, using the closed graph theorem (see also [11, Chapter I, Lemma 1.5]) we obtain

$$
\begin{aligned}
\int_{0}^{t} A \int_{0}^{\tau} T(s) x d s d \tau & =\int_{0}^{t} T(s) x d s-t x=A \int_{0}^{t} \int_{0}^{\tau} T(s) x d s d \tau \\
& =A \int_{0}^{t}(t-s) T(s) x d s .
\end{aligned}
$$

It follows that $\int_{0}^{t}(t-s) T(s) x d s$ does not always belong to $D\left(A^{2}\right)$, for this would imply that $D(A)=X$ and $A$ is bounded. It is then easy to see that the lemma does not hold for $\alpha>1$ (unless we are in the trivial case $D(A)=X$ ). However, one can prove by induction that

$$
x \in D\left(A^{n}\right) \Rightarrow \int_{0}^{t}(t-s)^{n} T(s) x d s \in D\left(A^{n+1}\right) .
$$

We are in a position to prove the following

Theorem 2.5. Assume that $E$ is a Banach space such that $D\left((-A)^{\alpha}\right) \subset E \hookrightarrow X$ for some $\alpha, 0<\alpha<1$. Then $A_{E}$ is the generator of a $\beta$-times integrated semigroup whenever $\beta>\alpha$.

Proof. We shall follow the proof of [5].

First of all, the family $U(t)=\Gamma(\beta)^{-1} \int_{0}^{t}(t-s)^{\beta-1} T(s) x d s, t \geq 0$, is a family of bounded linear operators on $D\left((-A)^{\alpha}\right)$ and $E$ by Lemma 2.4 and the closed graph theorem. We keep the same notation for the corresponding families in all the spaces $X, E$, and $D\left((-A)^{\alpha}\right)$. We shall denote the norm in $D\left((-A)^{\alpha}\right)$ 
by $\|\cdot\|_{\alpha}$. From the introduction, it follows that actually $D\left((-A)^{\alpha}\right) \hookrightarrow E \hookrightarrow X$. In the following calculations, $K$ will be a generic constant.

$$
\begin{aligned}
\Gamma(\beta)^{-1} & \|U(\tau) x\|_{E} \leq K\|U(\tau) x\|_{\alpha} \\
& =K\left\|(-A)^{\alpha} U(\tau) x\right\| \\
& =K\left\|s-\lim _{\varepsilon \rightarrow 0} \int_{\varepsilon}^{\infty} t^{-\alpha-1}(T(t)-I) U(\tau) x d t\right\| \\
& \leq K \int_{0}^{\infty} t^{-\alpha-1}\|(T(t)-I) U(\tau) x\| d t \\
& \leq K\left\{\int_{0}^{1} t^{-\alpha-1}\|(T(t)-I) U(\tau) x\| d t+\int_{1}^{\infty} t^{-\alpha-1}\|(T(t)-I) U(\tau) x\| d t\right\} \\
& \leq K\left\{\int_{0}^{1} t^{-\alpha-1} t^{\beta} d t+\int_{1}^{\infty} t^{-\alpha-1} d t \int_{0}^{\tau}(\tau-s)^{\beta-1} d s\right\}\|x\| \\
& =K\left(1+\tau^{\beta}\right)\|x\| .
\end{aligned}
$$

Hence, $\|U(\tau) x\|_{E} \leq K\left(1+\tau^{\beta}\right)\|x\| \leq K\left(1+\tau^{\beta}\right)\|x\|_{E}$.

In particular, the family $(U(t))_{t \geq 0}$ is exponentially bounded in $\mathscr{L}(E)$.

Continuity: $\|U(\tau) x-U(s) x\|_{E} \leq K\|U(\tau) x-U(s) x\|_{\alpha}$. Assume that $x \in$ $D\left((-A)^{\alpha}\right)$. Then it is clear that $(-A)^{\alpha} U(\tau) x=U(\tau)(-A)^{\alpha} x$. We further assume that $D\left((-A)^{\alpha}\right)$ is dense in $E$ (see Remark below). Then, by the BanachSteinhaus theorem, the family $(U(t))_{t \geq 0}$ is strongly continuous in $E$.

Generator: $(U(t))$ is a strongly continuous family on $X$ (the $\beta$-times integrated semigroup generated by $A$ ). In $X$ we have

$$
R(\lambda, A)=\lambda^{\beta} \int_{0}^{\infty} e^{-\lambda t} U(t) d t \text { for } \lambda>0
$$

If $x \in E$, the integral also converges in the topology of $E$ by the strong continuity and exponential boundedness proved above. Obviously $R(\lambda, A) E \subset E$ for all $\lambda \in \rho(A)$. By [5, Lemma 2.2], $\lambda \in \rho(A) \Rightarrow \lambda \in \rho\left(A_{E}\right)$ and $R(\lambda, A)_{\mid E}=$ $R\left(\lambda, A_{E}\right)$. It follows from Definition 2.1 that $(U(t))$ is the $\beta$-times integrated semigroup generated by $A_{E}$.

Remarks. (a) We have taken the norm in $D\left((-A)^{\alpha}\right)$ to be $\|x\|_{\alpha}=\left\|(-A)^{\alpha} x\right\|$, assuming implicitly that $A$ is invertible. Otherwise, the proof still works if we take the graph norm of $(-A)^{\alpha}$, namely $\|x\|+\left\|(-A)^{\alpha} x\right\|$.

(b) In the proof of strong continuity, we assumed that $D\left((-A)^{\alpha}\right)$ is dense in $E$. This assumption does not hold in the example presented in $\S 1$. In general, generators of $\beta$-times integrated semigroups are not densely defined. A proof in the general case can be given using Proposition 2.3 and the dominated convergence theorem.

Next, we want to extend Theorem 2.5 to the case $\alpha \geq 1$. First we note that we have the following formula which is a semigroup version of Taylor's formula:

$$
T(t) x=\sum_{p=0}^{n-1} \frac{t^{p}}{p !} A^{p} x+\int_{0}^{t} \frac{(t-s)^{n-1}}{(n-1) !} T(s) A^{n} x d s, \quad x \in D\left(A^{n}\right), \quad t \geq 0
$$


This formula may be obtained by induction from the remark following Lemma 2.4. If we assume that $0 \in \rho(A)$, then

$$
A^{-n} T(t) x=\sum_{p=0}^{n-1} \frac{t^{p}}{p !} A^{p-n} x+\int_{0}^{t} \frac{(t-s)^{n-1}}{(n-1) !} T(s) x d s, \quad x \in X, t \geq 0 .
$$

Note that the assumption $0 \in \rho(A)$ is not restrictive since perturbation by multiples of the identity operator preserves the generation property for integrated semigroups (see, e.g., Hieber [13] or Arendt, Neubrander, and Schlotterbeck [5]). We shall further make the assumption that $R(\lambda, A) E \subset E$ for $\lambda \in \rho(A)$.

Corollary 2.6. Let $n \geq 1$ and $0<\alpha<1$, and let $E$ be a Banach space such that $D\left((-A)^{n+\alpha}\right) \subset E \hookrightarrow X$. Assume that $R(\lambda, A) E \subset E$ for $\lambda \in \rho(A)$. Then $A_{E}$ generates an $(n+\beta)$-times integrated semigroup for $\beta>\alpha$.

Proof. Set

$$
S(t) x=\int_{0}^{t} \frac{(t-s)^{n+\beta-1}}{\Gamma(n+\beta)} T(s) x d s, \quad x \in X .
$$

From formula (2.6) we have

$$
A^{-n} \int_{0}^{t} \frac{(t-s)^{\beta-1}}{\Gamma(\beta)} T(s) x d s=S(t) x+\sum_{p=0}^{n-1} \frac{1}{p !} \int_{0}^{t} \frac{(t-s)^{\beta-1}}{\Gamma(\beta)} s^{p} d s A^{p-n} x
$$

which we rewrite as

$$
S(t) x=A^{-n} \int_{0}^{t} \frac{(t-s)^{\beta-1}}{\Gamma(\beta)} T(s) x d s-\sum_{p=0}^{n-1} \frac{1}{p !} \int_{0}^{t} \frac{(t-s)^{\beta-1}}{\Gamma(\beta)} s^{p} d s A^{p-n} x .
$$

From Lemma 2.4, we deduce that for $x \in X$,

$$
A^{-n} \int_{0}^{t} \frac{(t-s)^{\beta-1}}{\Gamma(\beta)} T(s) x d s \in D\left((-A)^{n+\alpha}\right)
$$

if $0<\alpha<\beta<1$. The condition $R(\lambda, A) E \subset E$ implies that $(S(t))$ is a family of bounded linear operators in $E$. The proof then continues as that of Theorem 2.5.

\section{SECOND-ORDER EQUATIONS}

In this section, we derive results analogous to those of the previous section for second-order linear differential equations. We have already recalled the fundamental facts on integrated cosine functions. In order to obtain interpolation results, we need here an analogue of Proposition 2.3 (see Hoppe [15, Theorem 2.7]). Let $C_{\alpha}:=\int_{0}^{\infty} t^{-2 \alpha-1}(\cos t-1) d t$.

Theorem 3.1. Assume that $A$ generates a uniformly bounded cosine function $(C(t))$ in $X$. Let $0<\alpha<1$ and $x \in X$. Then

$$
x \in D\left((-A)^{\alpha}\right) \text { if and only if } s-\lim _{\varepsilon \rightarrow 0} \frac{1}{C_{\alpha}} \int_{\varepsilon}^{\infty} t^{-2 \alpha-1}(C(t)-I) x d t \text { exists, }
$$

in which case it is equal to $-(-A)^{\alpha} x$. 
Taylor's formula for $C_{0}$-semigroups (formula (2.5)) has an equivalent for operator cosine functions (see Hoppe [15]):

$$
C(t) x=\sum_{p=0}^{n-1} \frac{t^{2 p}}{(2 p) !} A^{p} x+\int_{0}^{t} \frac{(t-s)^{2 n-1}}{(2 n-1) !} C(s) A^{n} x d s, \quad x \in D\left(A^{n}\right), \quad t \geq 0 .
$$

We have the following.

Lemma 3.2. Let $\tau \geq 0, x \in X$. We put $U_{\beta}(\tau) x:=\int_{0}^{\tau}(\tau-s)^{2 \beta-1} C(s) x d s$. Then if $0<\alpha<\beta \leq 1, U_{\beta}(\tau) x \in D\left((-A)^{\alpha}\right)$.

The proof is similar to that of Lemma 2.4, and we omit it.

From formula (3.2), we obtain

Proposition 3.3. Assume that $A$ generates a cosine function in $X$. Let $n \in$ $\mathbb{N}$, and let $E$ be a Banach space such that $D\left(A^{n}\right) \subset E \hookrightarrow X$. Assume that $R(\lambda, A) E \subset E$ for $\lambda \in \rho(A)$ (if $n>1)$. Then $A_{E}$ generates a $2 n$-times integrated cosine function in $E$.

This proposition was obtained in a slightly different formulation by Cioranescu and Henriquez [7]. Using Lemma 3.2 and the scheme of the proof of Theorem 2.5 and Corollary 2.6, we obtain the following.

Theorem 3.4. Let $n \geq 0$ and $0<\alpha<1$, and let $E$ be $a$ Banach space such that $D\left((-A)^{n+\alpha}\right) \subset E \hookrightarrow X$. If $n>1$, assume further that $R(\lambda, A) E \subset E$ for $\lambda \in \rho(A)$. Then $A_{E}$ generates a $2(n+\beta)$-times integrated cosine function in $E$ for $\beta>\alpha$.

Remark. One may ask if in Lemmas 2.4 and 3.2 the case $\alpha=\beta$ is admissible. This will have as a consequence that we also admit $\alpha=\beta$ in Theorem 2.5, Corollary 2.6, and Theorem 3.4. Actually, this is not always the case (see Fattorini $[10$, p. 86$]$ for a counterexample). In order that this should be true for cosine operator functions and for $\alpha=\frac{1}{2}$, Fattorini introduces the following condition $(F)$ :

(F) $\quad S(t) X \subset D\left((-A)^{1 / 2}\right)$ and $(-A)^{1 / 2} S(t)$ is strongly continuous

where $S(t) x=\int_{0}^{t} C(s) x d s, t \geq 0, x \in X$, is the sine function associated with $(C(t))$.

He then proves, using the Hilbert transform, that condition $(F)$ is always satisfied if $X=L^{p}(\Omega), 1<p<\infty$.

In the next section, we shall prove in a particular instance that one can admit $\alpha=\beta$ in the aforementioned theorems.

\section{Applications}

For the applications we have in view, we prove the following theorem. The ingredients of the proof are the spectral theorem and a classical estimate on the incomplete gamma function.

Theorem 4.1. Let $A$ be a normal operator in a Hilbert space $H$. Assume that $A$ is the generator of a uniformly bounded $C_{0}$-semigroup $(T(t))$. 
For $0<\alpha<1$, let $S(t) x=\int_{0}^{t}\left((t-s)^{\alpha-1} / \Gamma(\alpha)\right) T(s) x d s$. Then $S(t) H \subset$ $D\left((-A)^{\alpha}\right)$ and $(-A)^{\alpha} S(t)$ is strongly continuous.

Consequently, if $E$ is a Banach space with $D\left((-A)^{\alpha}\right) \subset E \hookrightarrow H$, then $A_{E}$ is the generator of an $\alpha$-times integrated semigroup.

Proof. Using the spectral theorem (multiplication operator-valued form), we may assume that $H=L^{2}(\Omega, \mu)$, where $(\Omega, \mu)$ is a measure space. Then (4.1)

$$
D(A)=\{f \in H, m \cdot f \in H\} \text { and } A f(x)=m(x) f(x) \text { for } f \in D(A),
$$

where $m: \Omega \rightarrow \mathbb{C}$ is a measurable function satisfying $\operatorname{Re}(m(x)) \leq 0$ almost everywhere in $\Omega$. It follows that $(T(t) f)(x)=e^{t \cdot m(x)} f(x), f \in H$. For $0<\alpha<1$

$$
\begin{aligned}
& D\left((-A)^{\alpha}\right)=\left\{f \in H,(-m(x))^{\alpha} \cdot f(x) \in H\right\}, \\
& (-A)^{\alpha} f(x)=(-m(x))^{\alpha} f(x) \text { for } f \in D\left((-A)^{\alpha}\right),
\end{aligned}
$$

where the determination of $(-m(x))^{\alpha}$ is conveniently chosen (see Fattorini [10, Chapter 3] for the definition of the fractional power).

Furthermore, the integrated semigroup $\Gamma(\alpha)^{-1} \int_{0}^{t}(t-s)^{\alpha-1} T(s) d s$ is the operator of multiplication by $\Gamma(\alpha)^{-1} \int_{0}^{t}(t-s)^{\alpha-1} e^{s m(x)} d s$. It remains to prove

$$
\underset{x \in \Omega}{\operatorname{ess}-\sup }\left|(-m(x))^{\alpha} \Gamma(\alpha)^{-1} \int_{0}^{t}(t-s)^{\alpha-1} e^{s m(x)} d s\right|<\infty
$$

$$
(-m(x))^{\alpha} \Gamma(\alpha)^{-1} \int_{0}^{t}(t-s)^{\alpha-1} e^{s m(x)} f(x) d s \text { is strongly continuous in } H .
$$

The estimate (4.3) follows from Gradshteyn and Ryzhik [12, formula 8.357, p. 942]. The continuity condition (4.4) then follows by the dominated convergence theorem.

We want to apply the preceding results to elliptic operators. First we fix some notations. Let $\Omega \subset \mathbb{R}^{N}$ be open and $\left(a_{i j}(x)\right), 1 \leq i, j \leq N$ be real-valued functions belonging to $W_{\mathrm{loc}}^{1,2}(\Omega)$ and such that $a_{i j}=a_{j i}$ (see [8, Chapter 1]). We assume that there are two positive constants $\lambda$ and $\mu$ such that the following ellipticity condition is satisfied.

$$
0<\lambda|\psi|^{2} \leq \sum_{1 \leq i, j \leq N} a_{i j}(x) \psi_{i} \overline{\psi_{j}} \leq \mu|\psi|^{2}, \quad \psi \in \mathbb{C}^{N} \backslash\{0\}, \text { a.e. in } \Omega \text {. }
$$

Consider the two nonnegative symmetric quadratic forms $a$ and $b$ :

$$
\begin{gathered}
a: H^{1}(\Omega) \times H^{1}(\Omega) \rightarrow \mathbb{C} \\
a(u, v)=\sum_{1 \leq i, j \leq N}\left(a_{i j}(x) \frac{\partial u}{\partial x_{i}}, \frac{\partial v}{\partial x_{j}}\right)_{L^{2}(\Omega)}
\end{gathered}
$$

and

$$
\begin{gathered}
b: H_{0}^{1}(\Omega) \times H_{0}^{1}(\Omega) \rightarrow \mathbb{C} \\
b(u, v)=\sum_{1 \leq i, j \leq N}\left(a_{i j}(x) \frac{\partial u}{\partial x_{i}}, \frac{\partial v}{\partial x_{j}}\right)_{L^{2}(\Omega)}
\end{gathered}
$$


Concerning the quadratic form $a$ and the associated operator, throughout we shall assume that $\Omega$ has a $C^{1}$-boundary. No regularity condition on $\Omega$ is required for $b$. Let $A_{2}^{N e}$ and $A_{2}^{D}$ be the maximal monotone operators associated with $a$ and $b$ respectively in $L^{2}(\Omega)$. Due to the symmetry assumption they are selfadjoint. Actually they are associated with the Neumann and Dirichlet boundary conditions respectively. The operators $-A_{2}^{N e}$ and $-A_{2}^{D}$ are generators of holomorphic semigroups of angle $\frac{\pi}{2}$ in $L^{2}(\Omega)$. They also generate uniformly bounded cosine operator functions (see also Fattorini [10]). We assume that $\Omega$ has bounded measure. Then for $p>2$ we denote by $A_{p}$ the realization of $A_{2}^{D}$ or $A_{2}^{N e}$ in $L^{p}(\Omega)$ and if $1<p<2$, we define $A_{p}$ as the adjoint of $A_{q}$ with $p^{-1}+q^{-1}=1$. For $p<\infty,-A_{p}$ is the generator of a holomorphic semigroup of angle $\frac{\pi}{2}$ in $L^{p}(\Omega)$. Under the above hypotheses, it was proved in Arendt [3] that if $\alpha=\frac{N}{2}\left|\frac{1}{2}-\frac{1}{p}\right|$, then $D\left(\left(-A_{2}\right)^{\alpha}\right) \subset L^{p}(\Omega)$ (compare with [8] where the case of $D\left((-A)^{1 / 2}\right)$ is treated). The proof of this result uses the Marcinkiewicz interpolation theorem. Combining this and Theorem 4.1 yields

Theorem 4.2. If $1<p<\infty$, the operator $i A_{p}$ is the generator of an $\alpha$-times integrated semigroup in $L^{p}(\Omega)$ for any $\alpha$ satisfying $\alpha \geq \frac{N}{2}\left|\frac{1}{2}-\frac{1}{p}\right|$.

This theorem was proved by Arendt [2] for $\alpha \in \mathbb{N}$ and for $\alpha \in \mathbb{R}, \alpha>$ $\frac{N}{2}\left|\frac{1}{2}-\frac{1}{p}\right|$, the result was established by El Mennaoui [9, Theorem 6.6, p. 52], using the Gaussian estimates on heat kernels from [8] and the Phragmén-Lindelöf theorem. Actually, El Mennaoui proves this as an application of a result of his on boundary values of holomorphic semigroups.

As for the wave equation we have the following result.

Theorem 4.3. Under the above assumptions, if $1<p<\infty$, the operator $-A_{p}$ is the generator of an $\alpha$-times integrated cosine function in $L^{p}(\Omega)$ for any $\alpha$ satisfying $\alpha \geq N\left|\frac{1}{2}-\frac{1}{p}\right|$.

This theorem has been considered by Cioranescu and Henriquez [7] for $\alpha \in$ $\mathbb{N}$.

\section{REFERENCES}

1. W. Arendt, Vector-valued Laplace transforms and Cauchy problems, Israel J. Math. 59 (1987), 327-352.

2. Sobolev imbeddings and integrated semigroups, 2nd International Conference on Trends in Semigroup Theory and Evolution Equations (Ph. Clément, E. Mitidieri, and B. de Pagter, eds.), Lecture Notes in Pure and Appl. Math., vol. 135, Marcel Dekker, New York, 1991.

3. —_ Cours de D.E.A. (Master's Level Course), Université de Franche-Comté, Besançon, 1993.

4. W. Arendt and H. Kellermann, Integrated solutions of Volterra integrodifferential equations and Cauchy problems, Integrodifferential Equations, Proc. Conf. Trento 1987 (G. Da Prato and M. Iannelli, eds.), Pitman Res. Notes Math. Ser., vol. 190, Longman Sci. Tech., Harlow, 1987, pp. 21-51.

5. W. Arendt, F. Neubrander, and U. Schlotterbeck, Interpolation of semigroups and integrated semigroups, Semigroup Forum 45 (1992), 26-37.

6. P. L. Butzer and H. Berens, Semigroups of operators and approximation, Springer-Verlag, New York, 1967. 
7. I. Cioranescu and H. Henriquez, Interpolation properties associated with second order differential equations, Aequationes Math. 47 (1994), 150-163.

8. E. B. Davies, Heat kernels and spectral theory, Cambridge Univ. Press, London, 1989.

9. O. El Mennaoui, Traces de semi-groupes holomorphes singuliers à l'origine et comportement asymptotique, Thèse, Besançon, 1992.

10. H. O. Fattorini, Second order linear differential equations in Banach spaces, North-Holland, Amsterdam, New York, and London, 1985.

11. J. A. Goldstein, Semigroups of linear operators and applications, Oxford Math. Monographs, Oxford Univ. Press, New York, 1985.

12. I. M. Gradshteyn and I. S. Ryzhik, Tables of integrals, series and products, Academic Press, New York, 1979.

13. M. Hieber, Integrated semigroups and differential operators on $L^{p}\left(\mathbb{R}^{N}\right)$-spaces, Math. Ann. 291 (1991), 1-16.

14. E. Hille and R. S, Phillips, Functional analysis and semigroups, Amer. Math. Soc. Colloq. Publ., vol. 31, Amer. Math. Soc., Providence, RI, 1957.

15. R. W. Hoppe, Interpolation of cosine operator functions, Ann. Mat. Pura Appl. (4) 136 (1984), 183-212.

16. H. Komatsu, Fractional powers of operators, Pacific J. Math. 19 (1966), 285-346.

17. F. Neubrander, Integrated semigroups and their applications to the abstract Cauchy problem, Pacific J. Math. 135 (1988), 111-155.

Equipe de Mathématiques, Universite de Franche-Comté, Route de Gray, 25030 Besancon Cedex, France

Current address: Department of Mathematics, University of Puerto Rico, Box 23355, San Juan, Puerto Rico 00931

E-mail address: v」keyantuo@upr1.upr.clu.edu 